\title{
Modelado del crecimiento de picaduras en tuberías enterradas que transportan hidrocarburos utilizando técnicas estadísticas ${ }^{(\bullet)}$
}

\author{
J.C. Velázquez ${ }^{*}{ }^{* *}$, F. Caleyo*, A. Valor ${ }^{* * *}$ y J.M. Hallen ${ }^{*}$
}

\begin{abstract}
Resumen
Esta investigación, resume los resultados de un estudio, enfocado en contribuir a resolver las necesidades de predicción de crecimiento de picadura en ductos que transportan hidrocarburos. Primero, se describe el desarrollo de un modelo determinista, considerando las propiedades físicas y químicas del suelo y el tipo de recubrimiento que protege la tubería. Este modelo fue utilizado para realizar simulaciones de Monte Carlo, logrando establecer las distribuciones de probabilidad de profundidad de picadura y su velocidad de crecimiento en suelos. Finalmente, se detalla el desarrollo de un modelo estocástico basado en cadenas de Markov. Este modelo tiene como objetivo, predecir la evolución en el tiempo de las distribuciones de profundidad de picadura.
\end{abstract}

\section{Pitting growth modelling in buried oil and gas pipelines using statistical techniques}

\begin{abstract}
New deterministic and stochastic predictive models are proposed for external pitting corrosion in underground pipelines. The deterministic model takes into consideration the local chemical and physical properties of the soil as well as the pipeline coating to predict the time dependence of pitting depth and rate in a range of soils. This model, based on results from a field study, was used to conduct Monte Carlo simulations that established the probability distribution of pitting depth and growth rate in the studied soils and their evolution over the life of the pipeline. In the last stage of the study, an empirical Markov chain-based stochastic model was developed for predicting the evolution of pitting corrosion depth and rate distributions from the observed properties of the soil.
\end{abstract}

Keywords

Pitting corrosion; Nonlinear regression; Monte Carlo simulations; Markov chains.

\section{INTRODUCCIÓN}

Mediante el uso de herramientas de inspección, es posible mantener en condiciones seguras de operación un ducto. No obstante, en algunas ocasiones no es posible realizar las inspecciones con la frecuencia necesaria. Por lo tanto, es necesario confiar en las estimaciones de las profundidades de defectos, basándose en inspecciones previas. Si hay un pronóstico erróneo de la severidad de estos defectos, se elaborará un programa de mantenimiento inadecuado. La estimación de la profundidad de picadura causada por corrosión en suelos, difícilmente puede ser cuantificada electroquímicamente. Esto se debe, a que en este fenómeno están involucradas una gran cantidad de variables, cuya sinergia obstaculiza la modelación mediante este enfoque.

Una de las primeras investigaciones basadas en análisis estadísticos, fue realizada por Romanoff $f^{11]}$. Este autor propuso un modelo general de crecimiento de picaduras, basado en la siguiente función:

$$
\mathrm{d}_{\max }(\mathrm{t})=\mathrm{kt}
$$

Donde: $d_{\max }(t)$, es la profundidad máxima de picadura, $t$, es el tiempo de exposición y "k", y "u", son variables a determinar mediante análisis de regresión.

\footnotetext{
(•) Trabajo recibido el día 03 de Septiembre de 2010 y aceptado en su forma final el día 20 de Diciembre de 2010.

* Departamento de Ingeniería Metalúrgica, ESIQIE, IPN, UPALM, EDIF. 7, Zacatenco, México D.F., 07738.

${ }^{* *}$ Faculty of Electrical Engineering, Mathematics and Computer Science, Delft University of Technology, Mekelweg 4, 2628 CD, Delft, The Netherlands.

*** Facultad de Física, Universidad de la Habana, San Lázaro y L. Vedado, 10400, La Habana, Cuba.
} 
En la presente investigación se modela la evolución del crecimiento de picaduras en tuberías de acero al carbono API-5L, utilizando un modelo determinista y un modelo estocástico. Ambos modelos consideran las propiedades físicas y químicas del suelo en contacto con el ducto estudiado, así como la protección catódica y recubrimiento.

Para realizar la modelación determinista, es necesario obtener un número significativo de valores de profundidades máximas de picadura en las secciones de tubo inspeccionadas, características del suelo, tiempos de servicio de la tubería, así como el tipo de recubrimiento. Posteriormente se realizó un análisis de regresión no lineal basado en la ecuación 1 .

Para conocer las distribuciones de probabilidad de la profundidad y rapidez de crecimiento de picaduras, se generan números aleatorios de las variables estudiadas.

Estos números se introducen en los modelos desarrollados en esta investigación, con el propósito de determinar la distribución de la profundidad o de la rapidez de crecimiento de defectos.

Una forma de modelar los fenómenos de naturaleza estocástica, es mediante cadenas de Markov. Tal es el caso del proceso de corrosión por picadura en ductos enterrados.

Esto se debe a que es posible discretizar el espesor de pared del tubo, en un número finito (o contable) de estados $[2$ y 3$]$.

\section{EXPERIMENTACIÓN}

Los datos utilizados en esta investigación fueron obtenidos de 259 muestras recolectadas en el sur de México. En cada sitio se midió la profundidad máxima de picadura en la sección de tubo. También se midieron algunas propiedades del suelo como: potencial redox, $\mathrm{pH}$, potencial suelo-tubo, resistividad, contenido de humedad, densidad aparente y contenido de iones. Las variables investigadas se muestran en la tabla I. Es importante mencionar, que la presente investigación fue llevada a cabo con financiamiento de los proyectos vinculados existentes entre el Instituto Politécnico Nacional y la empresa Petróleos Mexicanos. Por lo tanto, Petróleos Mexicanos determinó la cantidad de ductos a inspeccionar. En este contexto, para establecer la cantidad de muestras analizadas, no se tomó ningún criterio estadístico, solamente se propuso tener una cantidad de muestras suficientemente grande (>30) para asegurar la consistencia del modelo.

\section{RESULTADOS}

Los suelos analizados en la presente investigación, se clasificaron conforme al triángulo de texturas ${ }^{[4]}$ en arcillosos (110 muestras), franco-areno-arcillosos (79 muestras), franco-arcillosos (61 muestras), francolimo-arcillosos (6 muestras), limo-arcillosos (2 muestras) y franco-limosos (1 muestra). La textura del suelo fue definida en base a la relación entre arena, limo y $\operatorname{arcilla}^{[4]}$. El contenido mineral del suelo fue determinado por el hidrómetro Bouyoucos ${ }^{[5]}$. Como se puede observar, solo de tres tipos de suelo (arcilloso, franco-areno-arcilloso y franco-arcilloso), se tiene suficiente información como para analizarla estadísticamente por separado.

Los lugares donde se realizó el muestreo, no están en función del tipo de suelo, sino en zonas donde

Tabla I. Variables consideradas en el análisis

Table I. Investigated soil and pipe variables

\begin{tabular}{|c|c|c|c|}
\hline Variable & Símbolo & Unidades & Técnica de medición \\
\hline Profundidad máxima de picadura & $\mathrm{d}_{\mathrm{ma}}$ & $\mathrm{mm}$ & Micrómetro de profundidad \\
\hline Potencial redox & $\mathrm{rp}$ & $\mathrm{mV}^{*}$ & Medidor de potencial redox \\
\hline & $\mathrm{pH}$ & & Potenciómetro portátil \\
\hline Potencial suelo-tubo & $\mathrm{pp}$ & V & Electrodo de $\mathrm{Cu} / \mathrm{CuSO}_{4}$ \\
\hline Resistividad & re & $\mathrm{W}-\mathrm{m}$ & Método de Wenner \\
\hline Contenido de humedad & wC & $\%$ & Balanza termogravimétrica \\
\hline Densidad aparente del suelo & $\mathrm{bd}$ & $\mathrm{g} / \mathrm{ml}$ & Hidrómetro Bouyocos \\
\hline Contenido de iones cloruro & $\mathrm{CC}$ & ppm & Titulación volumétrica \\
\hline Contenido de iones bicarbonato & bc & ppm & Titulación volumétrica \\
\hline Contenido de iones sulfato & sc & $\mathrm{ppm}$ & Espectrofotómetro \\
\hline
\end{tabular}

* Respecto al electrodo estándar de hidrógeno. 
Petróleos Mexicanos y el Instituto Politécnico Nacional, considerando que pudiera presentarse deterioro del metal, debido a condiciones de trazo y perfil de la tubería. Consecuentemente, la cantidad de muestras por tipo de suelo, es el resultado de los criterios ingenieros y no de métodos estadísticos.

La tabla II muestra una descripción estadística de las variables analizadas de todas las muestras estudiadas. De los valores medios presentados en la tabla II, se puede inferir que la población de ductos investigada, tiene una edad promedio de 23 años aproximadamente. Se puede decir, que estos suelos son moderadamente corrosivos, ya que en promedio son ligeramente ácidos, con una resistividad relativamente alta (aprox. $50 \mathrm{~W}-\mathrm{m}$ ) y un potencial suelo tubo (natural o impuesto), de alrededor de $-0,85 \mathrm{~V}$, valor cercano al nivel óptimo de protección ${ }^{[6]}$.

En los estudios estadísticos realizados en la presente investigación, se observó que el 88 \% de los ductos estaban protegidos con algún tipo recubrimiento. Las condiciones de recubrimiento fueron categorizadas en cinco principales grupos: polímeros epóxicos (FBE), alquitrán de hulla, cinta poliolefínica, pintura asfaltada y tuberías sin recubrimiento.

Los proyectos de vinculación con Petróleos Mexicanos mencionados anteriormente, solamente consisten en determinar la integridad mecánica de los ductos. Por tal motivo, en la mayoría de los casos no fue posible medir el espesor de recubrimiento, debido a que alguna empresa realizó el servicio limpieza superficial de estos ductos.

Uno de los objetivos del presente trabajo fue conocer el efecto del tipo de suelo en el deterioro de los ductos. En este contexto, en la tabla III, se ilustra la muestra de la distribución de los recubrimientos para suelos arcillosos (A), franco-arcillosos (FA), franco-areno-arcillosos (FAA) y para todos los suelos (Todos). Se puede observar que casi el $12 \%$ de los ductos estudiados, no tenía recubrimiento al momento de ser inspeccionado, esto se debe en gran medida, a que la zona donde se encuentran estos ductos, es un área pantanosa. Esto provoca hundimientos, los cuales propician el desprendimiento de los recubrimientos. Aunque en un principio estos ductos pudieron haber estado recubiertos, no se sabe, en qué momento el recubrimiento perdió efectividad, por lo tanto se considera como una categoría independiente, llamada "tuberías sin recubrimiento".

A diferencia de otros modelos $^{[7}$ y 8$]$, el presente trabajo considera el recubrimiento como una variable independiente, a la cual se le asignaron valores de puntuación basándose en trabajos realizados pre-

Tabla II. Descripción estadística de las variables analizadas en todas las muestras de suelo

Table II. Statistics description at the analyzed variables

\begin{tabular}{|c|c|c|c|c|c|c|c|c|c|c|c|}
\hline & $\underset{(m m)}{d_{\max }}$ & $\underset{(m V)}{r p}$ & pH & $\begin{array}{l}\text { pp } \\
\text { (V) }\end{array}$ & $\begin{array}{c}\text { re } \\
(w-m)\end{array}$ & $\begin{array}{l}\text { wc } \\
(\%)\end{array}$ & $\begin{array}{l}\text { bd } \\
\text { (g/L) }\end{array}$ & $\begin{array}{c}c c \\
(p p m)\end{array}$ & $\begin{array}{c}\text { bc } \\
(\mathrm{ppm})\end{array}$ & $\begin{array}{c}\text { sc } \\
(\mathrm{ppm})\end{array}$ & $\begin{array}{c}t \\
\text { (años) }\end{array}$ \\
\hline Media & 2,02 & 167 & 6,13 & $-0,88$ & 50,2 & 23,9 & 1,30 & 47,7 & 19,6 & 153 & 22,90 \\
\hline Máximo & 13,44 & 348 & 9,88 & $-0,42$ & 399 & 66,0 & 1,56 & 672 & 195 & 1370 & 50,00 \\
\hline Mínimo & 0,41 & 2,14 & 4,14 & $-1,97$ & 1,91 & 8,7 & 1,10 & 0,0 & 0,0 & 0,0 & 5,00 \\
\hline
\end{tabular}

Tabla III. Puntaje asignado y frecuencia por tipo de recubrimiento

Table III. Coating type score and frequency by soil category

\begin{tabular}{lccccc}
\hline \multirow{2}{*}{ Tipo de recubrimiento (ct) } & Puntaje & \multicolumn{4}{l}{ Probabilidad asignada por tipo de suelo $\left(\mathbf{n} / \mathbf{n}_{\mathbf{T}}\right)$} \\
\cline { 3 - 6 } & & A & FA & FAA & Todos \\
\hline Sin recubrimiento & 1,0 & 0,102 & 0,119 & 0,194 & 0,118 \\
Pintura esmaltina & 0,9 & 0,019 & 0,017 & 0,039 & 0,024 \\
Cinta & 0,8 & 0,421 & 0,288 & 0,429 & 0,382 \\
Alquitrán & 0,7 & 0,449 & 0,542 & 0,273 & 0,443 \\
FBE & 0,3 & 0,009 & 0,034 & 0,065 & 0,033 \\
\hline
\end{tabular}

$n$ es el número de veces que se encontró el recubrimiento en mención y $n_{T}$ es el número total de ductos analizados. 
viamente ${ }^{[9}$ y 10$]$. Los valores de esta puntuación, también se presentan en la tabla III. Los valores más altos, son dados a recubrimientos con los más bajos rendimientos contra la corrosión.

\section{ANÁLISIS DE CORRELACIÓN}

Los resultados del análisis de correlación entre las variables estudiadas, se muestran en la tabla IV. Se puede observar la correlación media o poco significativa que existe entre cada variable. La matriz de correlación también tiene el propósito de evitar el uso de dos o más variables independientes, que estén fuertemente correlacionadas entre sí. La correlación de más peso ocurre entre el contenido de humedad y la resistividad. Un estudio realizado, hecho por Gupta ${ }^{[11]}$, confirmó esta correlación, mostrando que existe una correlación hasta un punto crítico, posteriormente la correlación entre humedad y resistividad deja de existir.

\section{DETECCIÓN DE VALORES ESPURIOS}

Un valor espurio, es una observación que se encuentra fuera del patrón de la distribución de datos analizados. Se utilizaron cuatro técnicas diferentes para detectar los valores espurios en el conjunto de datos medidos: (1) diagrama de caja, (2) residual estandarizado, (3) distancia de Cook y (4) matriz "Sombrero". Los datos que coinciden como espurios, en tres de las cuatro técnicas antes mencionadas, fueron considerados espurios. De los 259 datos colectados en el trabajo experimental, nueve fueron clasificados como espurios y por lo tanto fueron eliminados de la base de datos original.

\section{ELABORACIÓN DEL MODELO DETERMINISTA}

Los suelos analizados fueron recolectados en los estados mexicanos de Tabasco y Chiapas durante un periodo de tres años. El modelo propuesto en esta tesis, se basa en la ecuación (1). Sin embargo, se agrega un parámetro más a ajustar: el tiempo de iniciación de picadura $\left(\mathrm{t}_{\mathrm{sd}}\right)$ :

$$
d_{\text {max }}(t)=k\left(t-t_{s d}\right)^{v}
$$

El parámetro $t_{s d}$, se agrega al modelo, debido a que no se sabe el momento exacto en el cual comienza a crecer las picaduras que tendrán profundidad máxima en el futuro.

Basándose en la expresión (2), es posible mantener la no linealidad que caracteriza el crecimiento de picaduras. Los parámetros $k$ y $v$, se expresaron como funciones lineales de las variables independientes. Por lo tanto, la expresión (2) también se expresó de la siguiente forma:

$$
d_{\text {max }}(t)=\left(k_{0}+\sum_{i=1}^{n} k_{i} x_{i}\right)\left(t-t_{s d}\right)^{v_{0}+\sum_{j=1}^{m} v_{j} x_{j}}
$$

Donde: $x_{i}$, son las variables independientes, mientras $k_{i}$ y $v_{j}$, son parámetros de regresión.

Tanto para cada categoría de suelo, como para todas las observaciones, se realizó un análisis de regresión para las 1.024 combinaciones posibles, que resultan de distribuir las 10 variables independientes entre $k_{i}$ y $v_{\text {; }}$, bajo la condición, de que solo una variable puede ser asociada a cada parámetro. El modelo óptimo encontrado, fue aquel que tuvo mayor coeficiente de correlación $\left(\mathrm{R}^{2}\right)$.

Tabla IV. Matriz de correlación entre las variables analizadas

\begin{tabular}{|c|c|c|c|c|c|c|c|c|}
\hline & $\mathrm{pH}$ & pp & re & wc & bd & cc & bc & sc \\
\hline$r p$ & $-0,268$ & $-0,248$ & 0,339 & $-0,291$ & 0,05 & $-0,267$ & $-0,124$ & $-0,133$ \\
\hline pH & & 0,048 & $-0,320$ & 0,165 & 0,206 & $-0,051$ & 0,440 & 0,129 \\
\hline$p p$ & & & $-0,351$ & 0,274 & $-0,124$ & 0,302 & 0,038 & 0,158 \\
\hline re & & & & $-0,532$ & $-0,092$ & $-0,130$ & $-0,199$ & $-0,188$ \\
\hline wc & & & & & $-0,155$ & 0,196 & 0,117 & 0,193 \\
\hline bd & & & & & & $-0,229$ & 0,166 & 0,159 \\
\hline cc & & & & & & & 0,102 & 0,204 \\
\hline bc & & & & & & & & 0,268 \\
\hline
\end{tabular}

Table IV. Correlation matrix for independent variables 
El mejor modelo para todos los tipos de suelo ya mencionados, resultó aquel donde:

$$
\begin{gathered}
k_{i}=k_{0}+k_{r p} r p+k_{p H} p H+k_{r e} r e+ \\
k_{c c} c c+k_{b c} b c+k_{s c} s c \\
v_{j}=v_{0}+v_{p p} p p+v_{w c} w c+v_{b d} b d+v_{c t} c t
\end{gathered}
$$

Observando las expresiones (4) y (5), se puede afirmar que el vector parámetros $k_{i}$, es función del potencial redox, $\mathrm{pH}$, resistividad y contenido iónico. Por su parte, el vector de $v_{j}$, es función del potencial suelo - tubo, contenido de humedad, densidad del suelo y tipo de recubrimiento. Los resultados obtenidos del análisis de regresión, se muestran en la tabla V. En esta tabla se puede observar que los valores del coeficiente de correlación $\left(\mathrm{R}^{2}\right)$, son lo suficientemente altos como para hacer consistente la predicción del modelo.

También se puede observar, que los signos de correlación manifiestan adecuadamente la influencia de la variable correspondiente en el crecimiento de picadura. La profundidad máxima de picadura puede ser evaluada utilizando la expresión 3, con los valores correspondientes del coeficiente de correlación y se conocen las características físicas y químicas del suelo, que está en contacto con el tubo analizado.

\subsection{Análisis de sensibilidad}

Modelar el crecimiento de profundidad de picadura, es útil para determinar las variables que presentan mayor influencia en el proceso. La figura 1, muestra un gráfico de sensibilidad de las variables independientes, que presentan mayor influencia en la profundidad de picadura. En este gráfico, los valores de la pendiente, indican una mayor influencia de las variables independientes. El signo de esta pendiente indica la influencia directa o inversa de la variable en el crecimiento de picadura. De esta figura, se puede concluir que las variables de mayor influencia en el crecimiento de picadura son: el valor de $\mathrm{pH}$, el potencial suelo - tubo, el tipo de recubrimiento, la densidad aparente, el contenido de humedad y la concentración de iones cloruro. El resto de las variables tienen una influencia menor y no se presentan en la figura 1 .

Como se puede observar en la figura anterior, la resistividad, aparentemente no presenta una influencia significativa en el crecimiento de picadura en ductos enterrados.

Esto parece contradecir lo mencionado en la literatura. Sin embargo, se sabe que la resistividad está determinada por la humedad y el contenido iónico. Estas dos características si presentan una influencia significativa. Por lo tanto, si no se considera la humedad y el contenido iónico en el análisis de corrosividad del suelo, la resistividad jugaría un papel importante en la modelación.

\subsection{Ejemplo de aplicación}

El modelo propuesto puede ser aplicado al estudio de casos donde se desea realizar el estudio de integridad

\begin{tabular}{|c|c|c|c|c|}
\hline \multirow{2}{*}{ Parámetro } & \multicolumn{4}{|c|}{ Suelo/R ${ }^{2}$} \\
\hline & $\mathrm{A} / \mathbf{0 , 9 0}$ & FA/0,88 & FAA/0,71 & Todos/0,87 \\
\hline$k_{0}$ & 5,51E-01 & $9,84 \mathrm{E}-01$ & 5,99E-01 & 6,08E-01 \\
\hline$k_{0}$ & 8,85E-01 & $2,82 \mathrm{E}-01$ & $9,65 \mathrm{E}-01$ & 8,96E-01 \\
\hline$k_{r p}$ & $-8,98 \mathrm{E}-05$ & $-1,06 \mathrm{E}-04$ & $-1,82 \mathrm{E}-04$ & $-1,80 \mathrm{E}-04$ \\
\hline$k_{\mathrm{pH}}$ & $-5,90 \mathrm{E}-02$ & $-1,15 \mathrm{E}-01$ & $-6,42 \mathrm{E}-02$ & $-6,54 \mathrm{E}-02$ \\
\hline$k_{\mathrm{re}}^{\mathrm{pr}}$ & $-2,15 \mathrm{E}-04$ & $-2,99 \mathrm{E}-04$ & $-2,12 \mathrm{E}-04$ & $-2,60 \mathrm{E}-04$ \\
\hline $\mathbf{k}_{\mathrm{cc}}$ & 8,38E-04 & 1,80E-03 & 8,62E-04 & 8,74E-04 \\
\hline$k_{b c}$ & $-1,28 \mathrm{E}-03$ & $-4,88 \mathrm{E}-04$ & $-6,78 \mathrm{E}-04$ & $-6,39 \mathrm{E}-04$ \\
\hline$k_{s c}$ & $-5,33 \mathrm{E}-05$ & $-2,09 \mathrm{E}-04$ & $-1,13 \mathrm{E}-04$ & $-1,22 \mathrm{E}-04$ \\
\hline$v_{p p}$ & 4,93E-01 & 4,61E-01 & 5,12E-01 & 5,19E-01 \\
\hline$v_{w c}$ & 3,72E-03 & 1,69E-02 & 4,50E-03 & 4,65E-03 \\
\hline$v_{b d}$ & -1,01E-01 & -9,87E-02 & $-1,58 \mathrm{E}-01$ & $-9,90 \mathrm{E}-02$ \\
\hline$v_{c t}$ & 4,67E-01 & 5,67E-01 & 4,34E-01 & 4,31E-01 \\
\hline$t_{s d}$ (años) & 3,05 & 3,06 & 2,57 & 2,88 \\
\hline
\end{tabular}

Tabla V. Resultados del análisis de regresión

Table V. Result of the regression analysis 


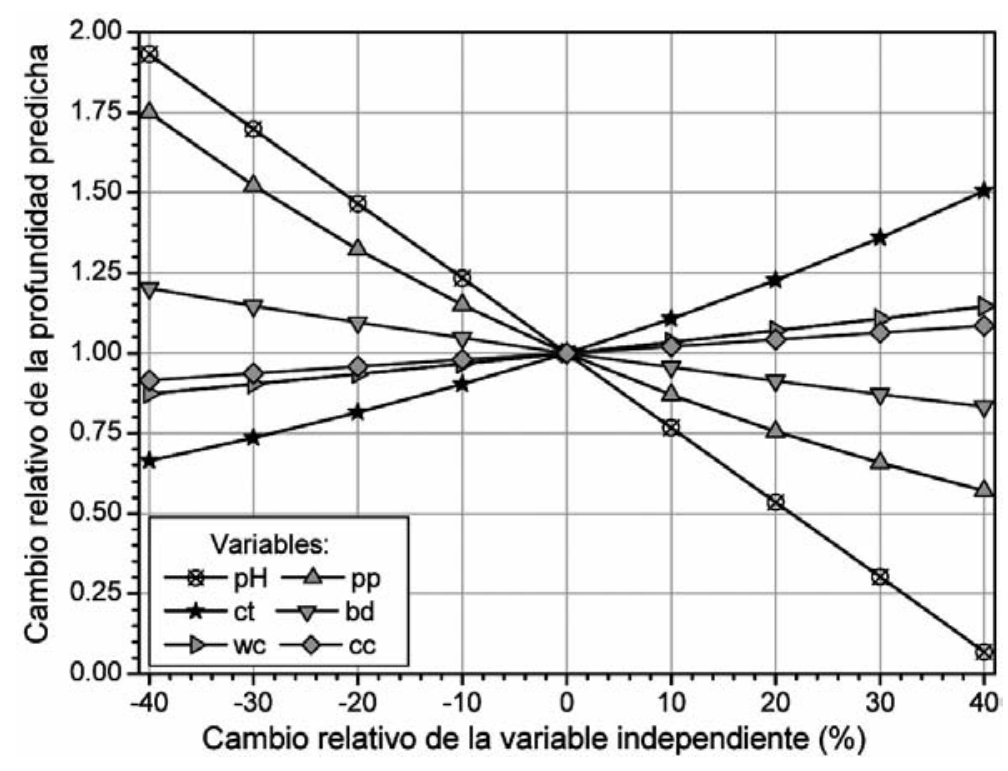

Figura 1. Análisis de sensibilidad del modelo.

Figure 1. Model sensitivity analysis.

de ductos. En la presente investigación, se aplicó a un ducto de $914 \mathrm{~mm}$ de diámetro y un espesor de pared de $11 \mathrm{~mm}$. Este ducto, fue puesto en operación en 1979, e inspeccionado en 1997, utilizando inspección en línea. Con el propósito de comparar las dos formas de estimar la profundidad de picaduras, primero se realizó una evaluación, asumiendo que la evolución en el tiempo de la profundidad de picadura, muestra un comportamiento lineal. Esto también hace suponer, que la velocidad de crecimiento es constante en el tiempo. Esto significa que la profundidad del defecto para cualquier tiempo, puede ser estimada mediante la siguiente expresión:

$$
d_{c r}(t)=v c\left(t-t_{1}\right)
$$

Donde $t_{I}$, es el tiempo en el que se realizó la inspección; el subíndice cr, indica que la predicción es realizada suponiendo la velocidad de crecimiento constante; $v c$ y $t$, es el tiempo para el cual se realiza la estimación. La velocidad de crecimiento constante se calcula de la siguiente forma:

$$
v c=\frac{d_{m}}{t_{1}-t_{c}}
$$

Donde $d_{m}$, es la profundidad medida y $t_{I}-t_{c}$, es el intervalo de tiempo entre la inspección y la construcción del ducto.

El tiempo de falla por fuga en el ducto $\left(t_{L}\right)$, puede ser determinado igualando $d_{c r}(t)$ al espesor de pared y resolviendo $t$ en la igualdad resultante. En estudios de ingeniería, las expresiones 6 y 7 , son utilizadas comúnmente para predecir el tiempo de falla.

En el ducto estudiado, se detectaron 123 picaduras en la superficie externa.

Posteriormente, se recopiló información de la profundidad de picadura medida en campo, en el momento de reparar las secciones que comprometían la integridad mecánica de la línea. Las profundidades de picaduras medidas en campo, fueron comparadas con las predichas mediante las expresiones 6 y 7 . Esta comparación se muestra en la figura 2 . Se puede observar que existe un sesgo en los puntos de comparación, que denotan una sobreestimación de la profundidad predicha respecto a la profundidad real medida en campo. Esto significa, que utilizando el modelo de crecimiento lineal, se recomendaría la reparación de tramos del ducto antes de lo necesario.

Para mejorar el resultado mostrado en la figura anterior, se propuso aplicar el modelo desarrollado en la presente investigación. Derivando la ecuación (2), se tiene, que la velocidad de crecimiento de picadura puede ser expresada de la siguiente forma:

$$
v c(t)=k v\left(t-t_{s d}\right)^{-1}
$$

Para predecir la profundidad de defectos, la expresión anterior fue evaluada con los valores de los parámetros $\left(k_{i} y v_{j}\right)$, para la categoría que incluye todos los suelos. De esta forma, el crecimiento de picadura para tiempos posteriores, fue determinado de la siguiente manera: 


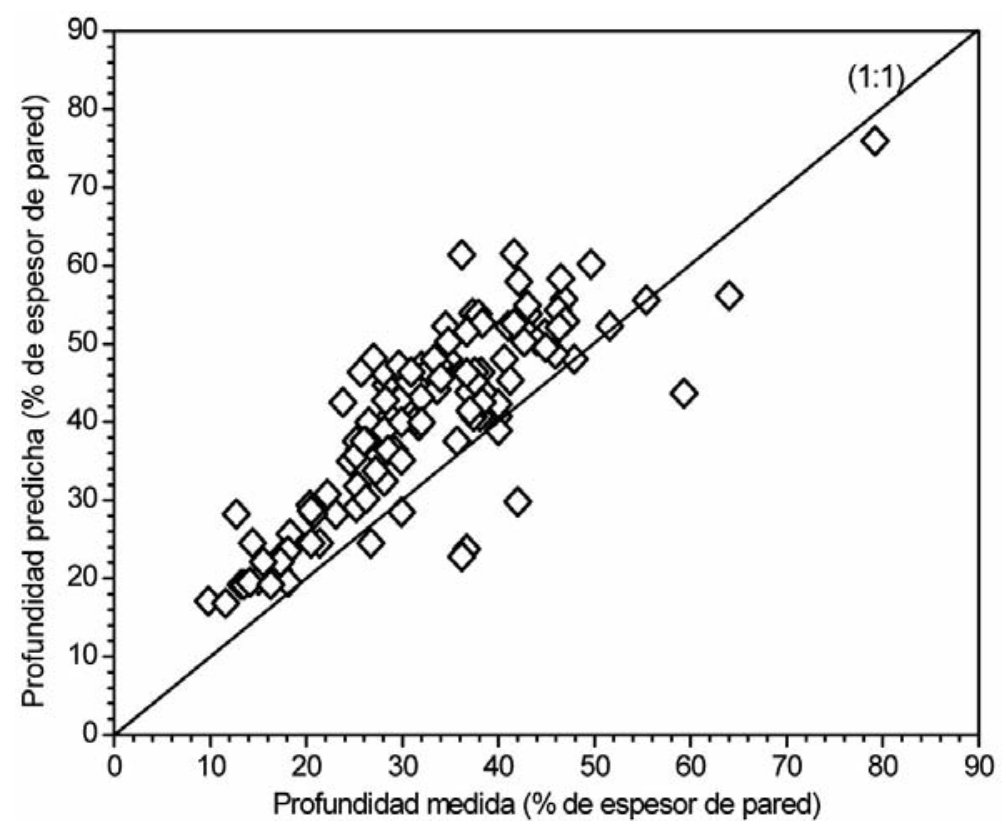

Figura 2. Profundidad de picadura predicha utilizando el modelo no lineal contra profundidad real.

Figure 2. Pit depth predicted using the linear pit growth model against actual depth.

$$
d(t)=d_{m}+\int_{t_{1}}^{t} v c_{\text {todos }}\left(t^{\prime}\right) d t^{\prime}
$$

Donde $v c(t)$, es la velocidad de crecimiento (en $\mathrm{mm} / \mathrm{año}$ ) y $t^{\prime}$, es una variable ficticia. El subíndice "todos", significa que se utilizaron los parámetros correspondientes a esa categoría.

Los resultados de la aplicación, del modelo propuesto en este trabajo, se muestran en la figura 3. Se puede observar que el sesgo de las estimaciones hacia profundidades superiores a las reales, se ha reducido considerablemente. El sesgo y la dispersión de los resultados, pueden atribuirse al hecho, de que se utilizó el modelo que incluye los parámetros, en condiciones promedio de la categoría de todos los suelos. Si se utilizara el modelo con las características físicas y químicas de cada sitio, se podría obtener un mejor resultado con menor sesgo e incertidumbre.

\section{ESTUDIO DE LA DISTRIBUCIÓN DE LA PROFUNDIDAD DE PICADURA Y SU VELOCIDAD DE CRECIMIENTO}

En la literatura, es frecuente encontrar diferentes distribuciones de probabilidad, que representen la profundidad de picadura y su velocidad de crecimiento.
Entre las distribuciones que ajustan a las profundidades máximas de picadura, están: la distribución Log-Normal ${ }^{[8]}$, la distribución Gamma Generalizada $^{[8]}$, y la distribución de Gumbe ${ }^{[12]}$, Weibull[13] y Fréchet ${ }^{[12]}$. Sin embargo, surge la pregunta: -iCuál es la que mejor representa la profundidad y crecimiento de picadura?-. Para responder esta pregunta, el presente estudio utiliza la técnica de simulaciones de Monte Carlo y los modelos deterministas presentados anteriormente.

\subsection{Simulaciones de Monte Carlo}

El método de Monte Carlo es un método probabilista, que es utilizado cuando no es posible calcular un resultado exacto mediante un algoritmo determinista $^{[14]}$. Este método consiste en generar números aleatorios de las variables de entrada, de un modelo a partir de las distribuciones de probabilidad conocidas para estas variables. Para generar estos valores aleatorios, se realiza una transformación inversa de la función de distribución acumulativa. Para una variable aleatoria $x$, una realización aleatoria de la misma, que responda a una distribución $F$, se puede obtener generando un número $u$, distribuido uniformemente entre 0 y 1 y resolviendo $x=F^{-1}(u)^{[14]}$. 


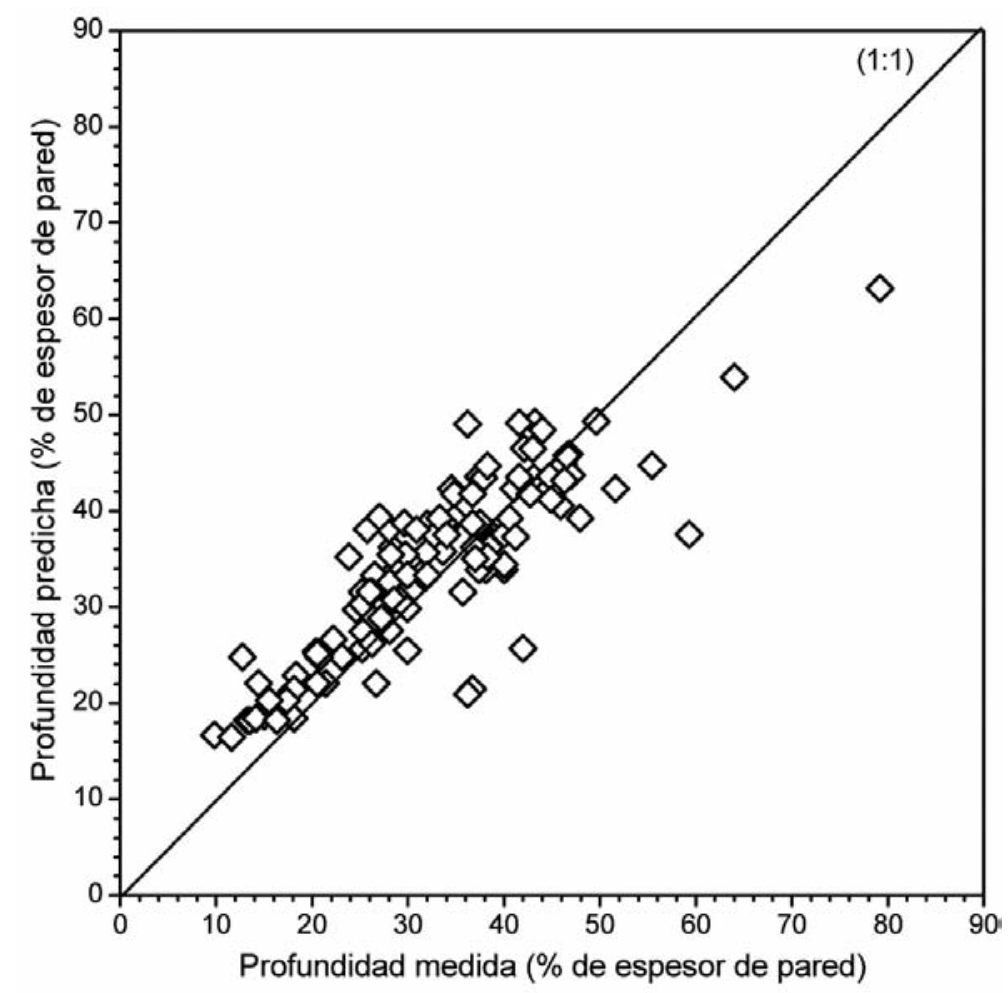

Figura 3. Comparación de la profundidad predicha (utilizando (9)) y profundidad medida.

Figure 3. Comparison between predicted pit depth (using ecuation (9)) and actual pit depth.

Para aplicar esta técnica, es necesario contar con modelos de tipo determinista, que representen la evolución en el tiempo de la profundidad de picadura y la velocidad de crecimiento. También es necesario conocer las distribuciones de probabilidad asociadas a las variables independientes que alimentan estos modelos. Para conocer estas distribuciones, se utilizaron los datos obtenidos durante las mediciones en campo de las propiedades del suelo y el tipo de recubrimiento. De la información recopilada se procedió a ajustar el conjunto de datos de cada variable, a diferentes distribuciones de probabilidad. De estos ajustes se seleccionó el mejor, basándose en la prueba de Kolmogorov-Smirnov ${ }^{[15]}$. Los resultados obtenidos de estos ajustes se muestran en la tabla VI.

\subsection{Resultados y discusión de la simulación de profundidad de picadura}

La evolución en el tiempo de la media y la variante (no paramétricas) de la distribución de profundidad máxima de picadura por tipo de suelo, se muestran en las figu- ras 4 y 5 respectivamente. De estas figuras se puede observar que tanto la media, como la variante de la distribución, aumentan conforme aumenta el tiempo. En el caso de la evolución de la media, se confirma que el proceso de crecimiento de picadura, tiene una conducta ligeramente no lineal. Para el caso de la variante, los resultados de la evolución confirman el carácter estocástico del proceso. Esto puede afirmarse debido a que la dispersión de los valores de velocidad de crecimiento simulados, aumenta conforme aumenta el tiempo.

En la figura 5 también se puede observar, que los suelos más corrosivos presentan mayor variante. Esto se debe a que los suelos más corrosivos, como en el caso de los suelos arcillosos, presentan mayor dispersión las variables independientes utilizadas en las predicciones.

Otra información importante que es posible obtener de las simulaciones de Monte Carlo de profundidad máxima de picadura, es el tipo de distribución (Weibull, Gumbel o Fréchet) que mejor representa este fenómeno. Por tal motivo, en la presente investigación se hace uso de la distribución generalizada de valores extremos (GEVD, por sus siglas en inglés).

En probabilidad y estadística, GEVD es una familia de distribuciones continuas de probabilidad. Esta 
Tabla VI. Distribuciones de probabilidad ajustadas a los datos en formato (media, variante)

Table VI. Statistical fitting of the observed corrosion data expressed in mean and variance format

\begin{tabular}{|c|c|c|c|c|}
\hline \multirow{2}{*}{$\begin{array}{l}\text { Variable } \\
\text { (unidades) }\end{array}$} & \multicolumn{4}{|c|}{ Tipo de suelo } \\
\hline & A & FA & FAA & Todos \\
\hline$r e(w-m)$ & Weibull $(62,2 ; 4.275)$ & Weibull $(28,2 ; 566)$ & Lgnormal $(49,2 ; 2.363)$ & Lgnormal $(49,8 ; 2.931)$ \\
\hline$s c(p p m)$ & Gamma $(131 ; 12.566)$ & Lgnormal $(208 ; 65.549)$ & Weibull $(144 ; 9.836)$ & Lgnormal $(154 ; 25.328)$ \\
\hline$b c(p p m)$ & Lgnormal $(19.4 ; 639)$ & Lgnormal $(23,2 ; 548)$ & Lgnormal $(13,8 ; 36,1)$ & Lgnormal $(18,6 ; 436)$ \\
\hline$c c(p p m)$ & Lgnormal $(53,2 ; 4.709)$ & Lgnormal $(45,0 ; 2.946)$ & Lgnormal $(21,82 ; 559)$ & Lgnormal $(41,3 ; 3.135)$ \\
\hline$w c(\%)$ & Normal $(24,0 ; 47,2)$ & Weibull $(24,7 ; 27,07)$ & Normal $(22,4 ; 32,80)$ & Normal $(23,7 ; 38,3)$ \\
\hline ph & Gumbel $(5,94 ; 0,97)$ & Gumbel $(6,36,0,77)$ & Normal $(6,23 ; 0,637)$ & Gumbel $(6,13 ; 0 \mathrm{M} 84)$ \\
\hline$p p(V)$ & Normal $(-0,86 ; 0,04)$ & Normal $(-0,81 ; 0,037)$ & Normal $(-0,92 ; 0,023)$ & Normal $(-0,86 ; 0,04)$ \\
\hline$b d(\mathrm{~g} / \mathrm{ml})$ & Normal $(1,22 ; 2,8 \mathrm{E}-3)$ & Gumbel $(1,32 ; 5,0 \mathrm{E}-4)$ & Gumbel $(1,39 ; 2,2 \mathrm{E}-3$ & Normal $(1,30 ; 7,4 \mathrm{E}-3)$ \\
\hline$r p(\mathrm{mV})$ & Uniforme $(2,14 ; 348)^{2}$ & Uniforme $(19,0 ; 301)$ & Uniforme $(20,0 ; 339)$ & Uniforme $(2,14 ; 348)$ \\
\hline
\end{tabular}

${ }^{1}$ GEVD: Distribución generalizada de valores extremos.

${ }^{2}$ Para la distribución uniforme, el rango está dado por los dos primeros momentos.

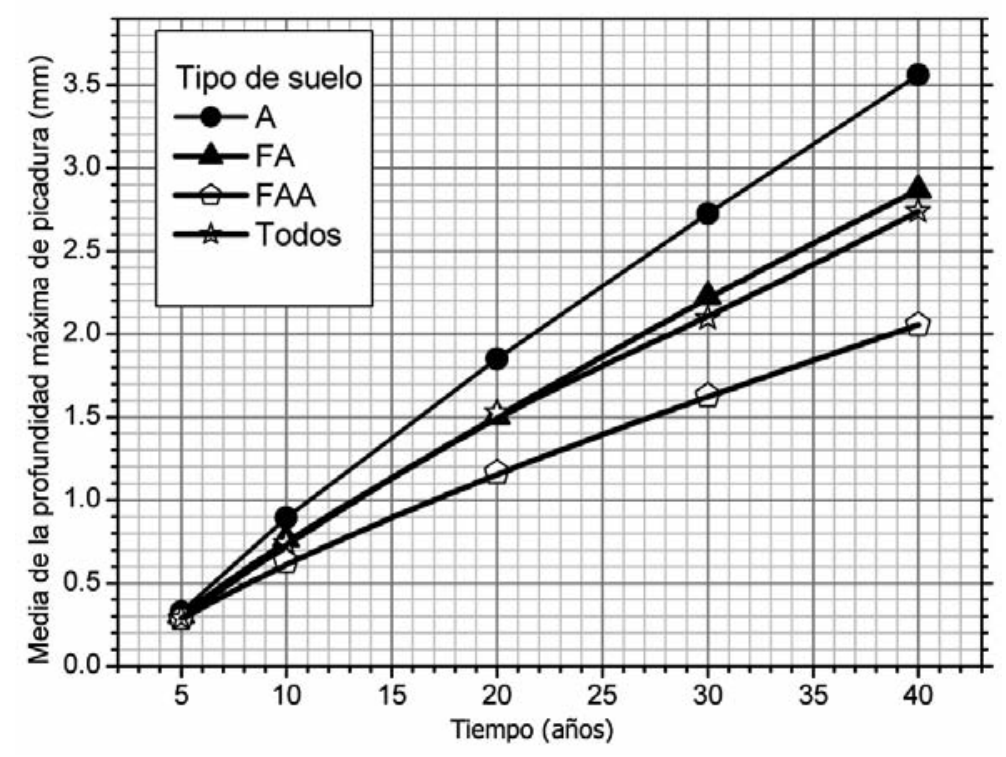

Figura 4. Evolución de la media de los valores de profundidad máxima de picadura.

Figure 4. Evolution of the mean of the maximum pit depth.

función de distribución fue desarrollada dentro de la teoría de valores extremos para combinar las distribuciones de Gumbel (Tipo I), Fréchet (Tipo II) y Weibull (Tipo III). La función de probabilidad acumulativa (CDF, por sus siglas en inglés) de la Distribución Generalizada de Valores Extremos, es mostrada a continuación ${ }^{[15]}$ :

$$
F(x)=\exp \left(-(1+\xi z)^{-1 / \xi}\right)
$$

Donde: $z=\frac{x-b}{a}$ es una variable reducida, es el parámetro de forma, es el parámetro de localización, es el parámetro de escala $y, x$, es la variable aleatoria. 


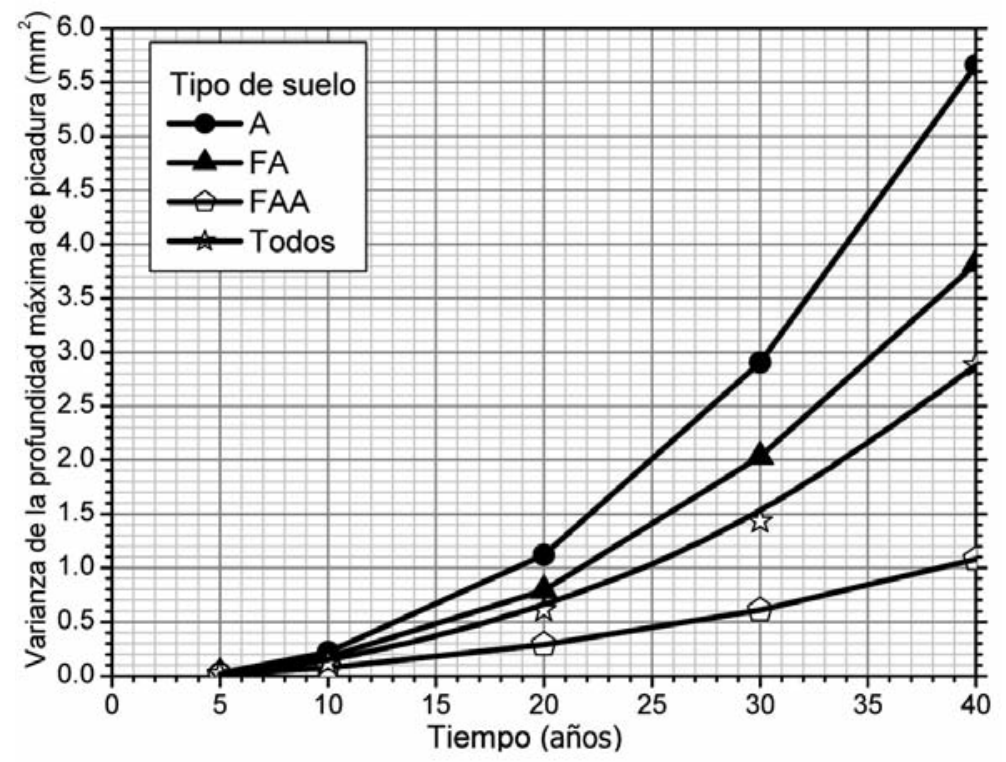

Figura 5. Evolución de la variante de los valores de profundidad máxima de picadura.

Figure 5. Evolution of the variance of the maximum pit depth.

La figura 6, muestra como el valor del parámetro de forma $(\xi)$ de GEVD ajustada, se incrementa conforme avanza el tiempo. Es decir, en ningún tipo de suelo, este parámetro permanece constante en el tiempo.
La figura 6, muestra como para tiempos de exposición relativamente pequeños, $(<15$ años), la distribución de Weibull es más apropiada. Por otra parte, para tiempos de exposición relativamente grandes (> 15 años), las distribuciones de Gumbel y Fréchet

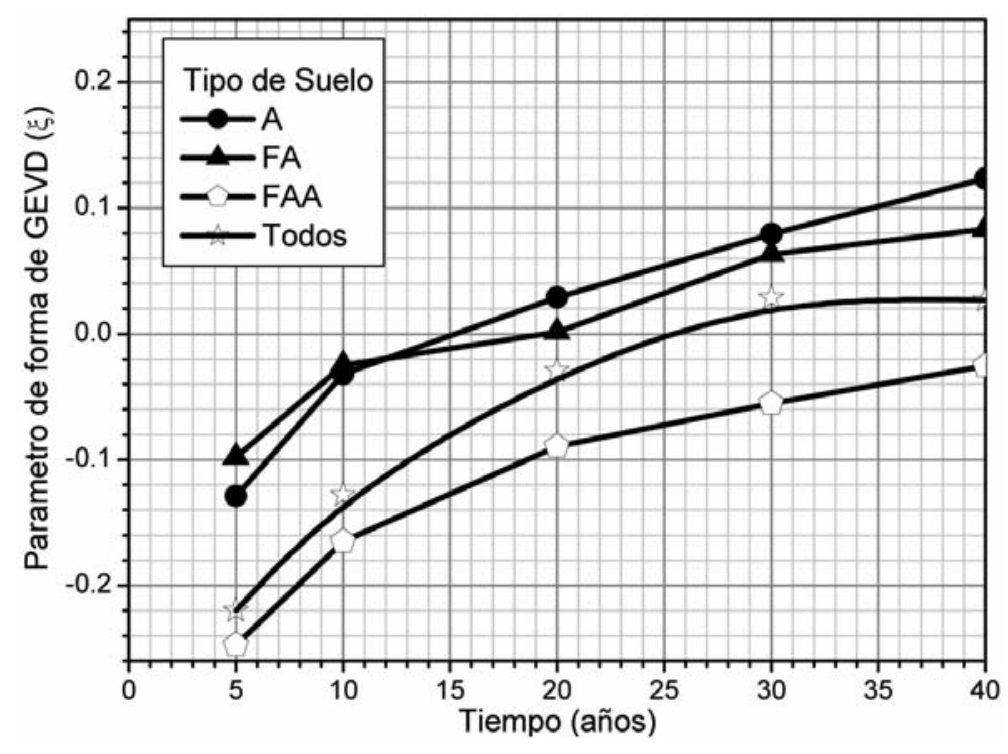

Figura 6. Evolución del parámetro de forma $(\xi)$ de la distribución de la profundidad depicadura.

Figure 6. Evolution of the shape parameter $(\xi)$ of the pit depth distribution. 
resultan las más apropiadas. De las figuras 7 y 8 , se puede notar que es evidente el cambio en la forma de la distribución de profundidad máxima de picadura, ya sea por tipo de suelo o por tiempo de exposición. Mostrándose un cambio en las características leptocurticas de la distribución y un sesgo hacia los valores positivos. Por ejemplo, en la figura 7 se observa que "las colas" de las distribuciones de profundidad de picadura a tiempos prolongados, son más extensas.

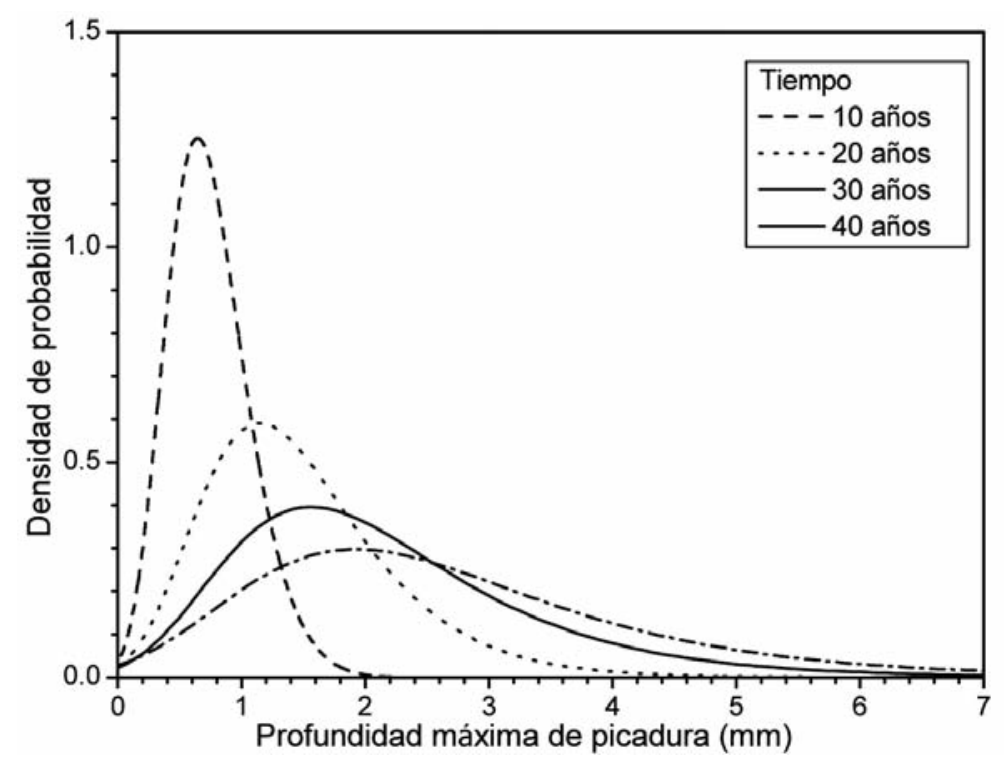

Figura 7. Ajuste de datos obtenidos en las simulaciones utilizando datos de todos los suelos.

Figure 7. Fit to the all soils data for different exposure times for all soils category.

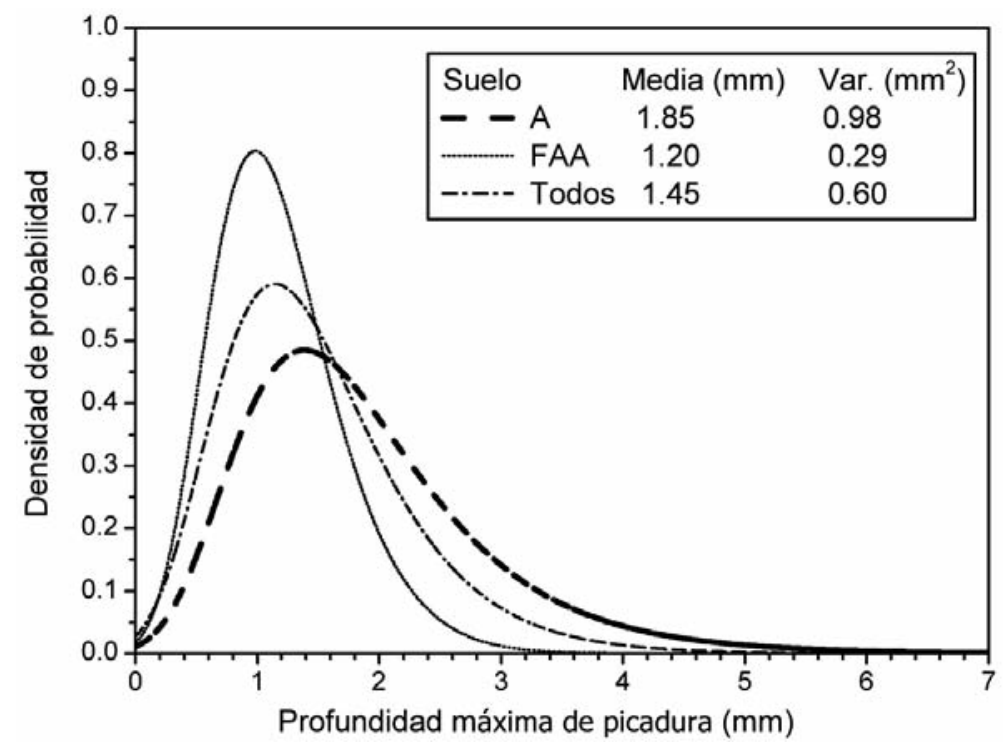

Figura 8. Ajuste a GEVD de datos obtenidos en las simulaciones a 20 años.

Figure 8. Fit to GEVD of simulated pit depth in 20 years exposure. 


\subsection{Dependencia del tiempo de la distribución de velocidad de crecimiento de picadura}

Así como en el caso de las profundidades máximas de picadura, las figuras 9 y 10 presentan respectivamente la evolución en el tiempo de la estimación no paramétrica de la media y la variante de las distribuciones de velocidad de crecimiento simuladas.

Los resultados obtenidos por la figura 10 , indican que la variante de la velocidad de crecimiento, también muestra una tendencia a disminuir conforme se

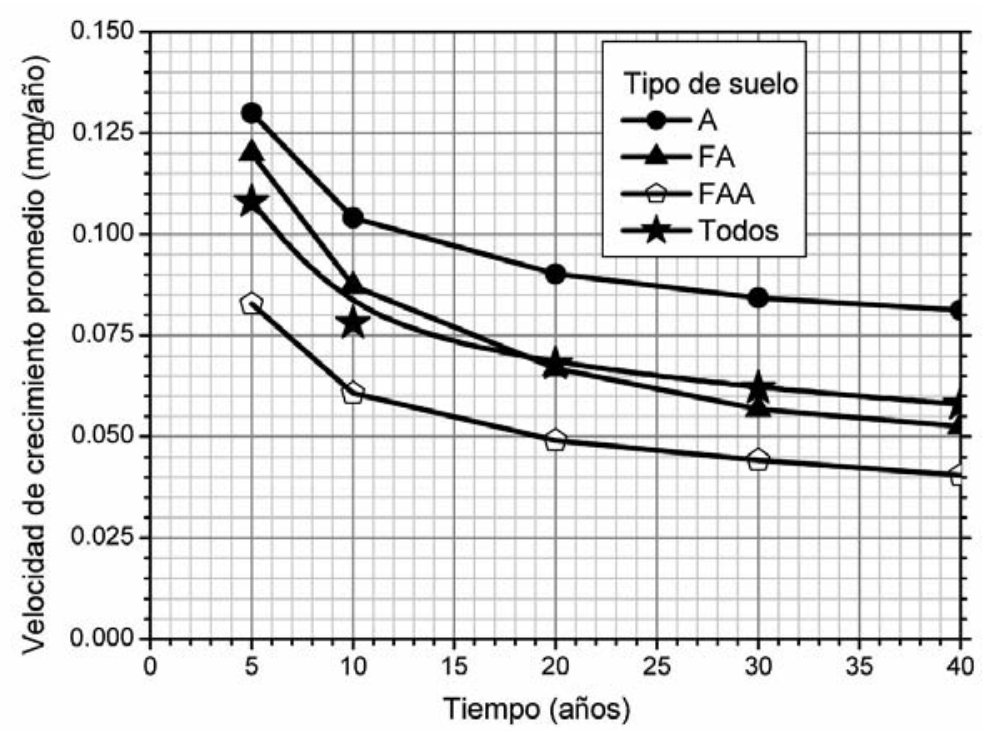

Figura 9. Evolución de la media de la distribución de velocidad de crecimiento de picadura.

Figure 9. Evolution of the mean of the pitting corrosion rate.

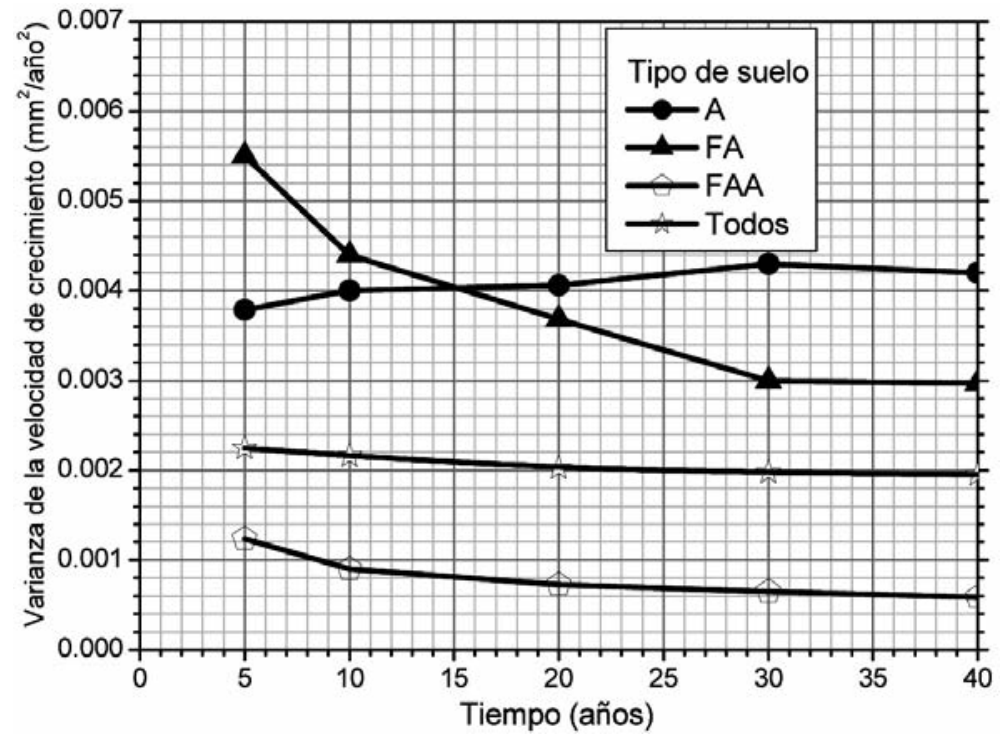

Figura 10. Evolución de la variante de la distribución de velocidad de crecimiento de picadura.

Figure 10. Evolution of the variance of the pitting corrosion rate. 
incrementa el tiempo, para luego permanecer relativamente constante a tiempos relativamente prolongados (> 30 años), en todos los tipos de suelo estudiados. Esto puede ser interpretado como un indicio de que, no solamente la media, sino también la variante de la velocidad de crecimiento, es mayor para picaduras que acaban de nacer, que para picaduras con un tiempo de vida mayor. El mismo se explica por el hecho de que a tiempos relativamente grandes, las picaduras más profundas tienen más probabilidad de crecer y este crecimiento ocurre con una velocidad aparentemente constante, debido a que se alcanza el periodo de estabilidad, en el cual el crecimiento de picadura, es gobernado por el proceso de difusión de las especies iónicas involucradas ${ }^{[16]}$.

La figura 11, ilustra la evolución en el tiempo del parámetro, de forma de la Distribución Generalizada de Valores Extremos, ajustada a los datos simulados de la velocidad de crecimiento. Como en el caso de la distribución de profundidad máxima de picadura, las distribuciones de Weibull, Gumbel y Fréchet, pueden describir al fenómeno, dependiendo del periodo de tiempo que se estudie.

De los resultados obtenidos en estas simulaciones, se puede observar que las picaduras más profundas son las que tienen más probabilidad de seguir creciendo, pero disminuyendo su velocidad de crecimiento conforme avanza el tiempo.

\subsection{Aplicación}

En esta sección se presenta un caso donde se estima el estado de un ducto en operación que transporta gas natural. Este ducto es de aproximadamente $82 \mathrm{~km}$ de longitud y está protegido con alquitrán de hulla. El diámetro exterior es de 355,6 $\mathrm{mm}$ y tiene un espesor de pared de $9,52 \mathrm{~mm}$. Este ducto fue puesto en operación en 1981. Las inspecciones en línea de este ducto, fueron llevadas a cabo en los años 2002 y 2007. El ducto está protegido catódicamente. Las distribuciones de la profundidad de defectos, se muestran en las figuras 12 y 13 para las inspecciones de 2002 y 2007 respectivamente.

Para llevar a cabo las estimaciones, se partió de la suposición de que el suelo en contacto con el tubo, tenía las mismas características que la categoría de "todos los suelos". En este contexto, se asumió que la distribución de velocidad de crecimiento de defectos $\left(g(v)_{t}\right)$, está representada por la distribución correspondiente a 20 años. Esta distribución se utilizó para estimar la distribución de los defectos, a partir de la distribución de defectos medidos en el $2002\left(f(x)_{02}\right)$, para así conocer la distribución en $2007\left(f(x)_{07}\right)$.

La aproximación fue hecha mediante la siguiente expresión:

$$
f(x)_{07}=\int_{0}^{\infty} g(v)_{t} f_{i l}(x-v \delta)_{02} d v
$$

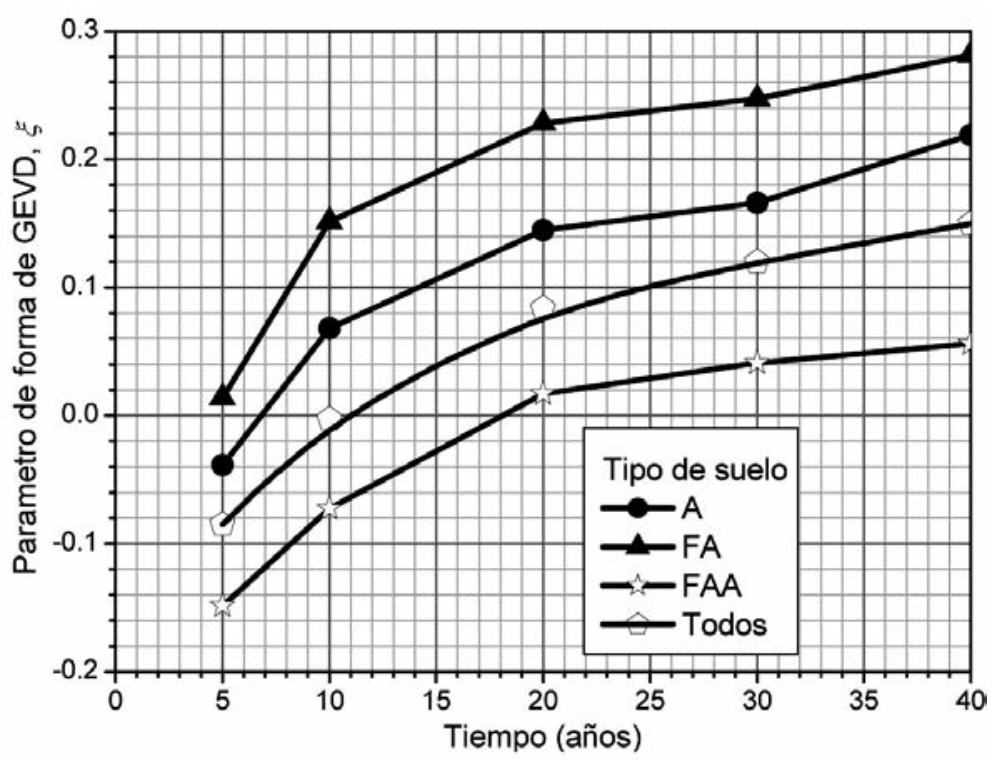

Figura 11. Evolución del parámetro de forma de la distribución de velocidad de crecimiento.

Figure 11. Evolution of the shape parameter of the pitting corrosion rate. 


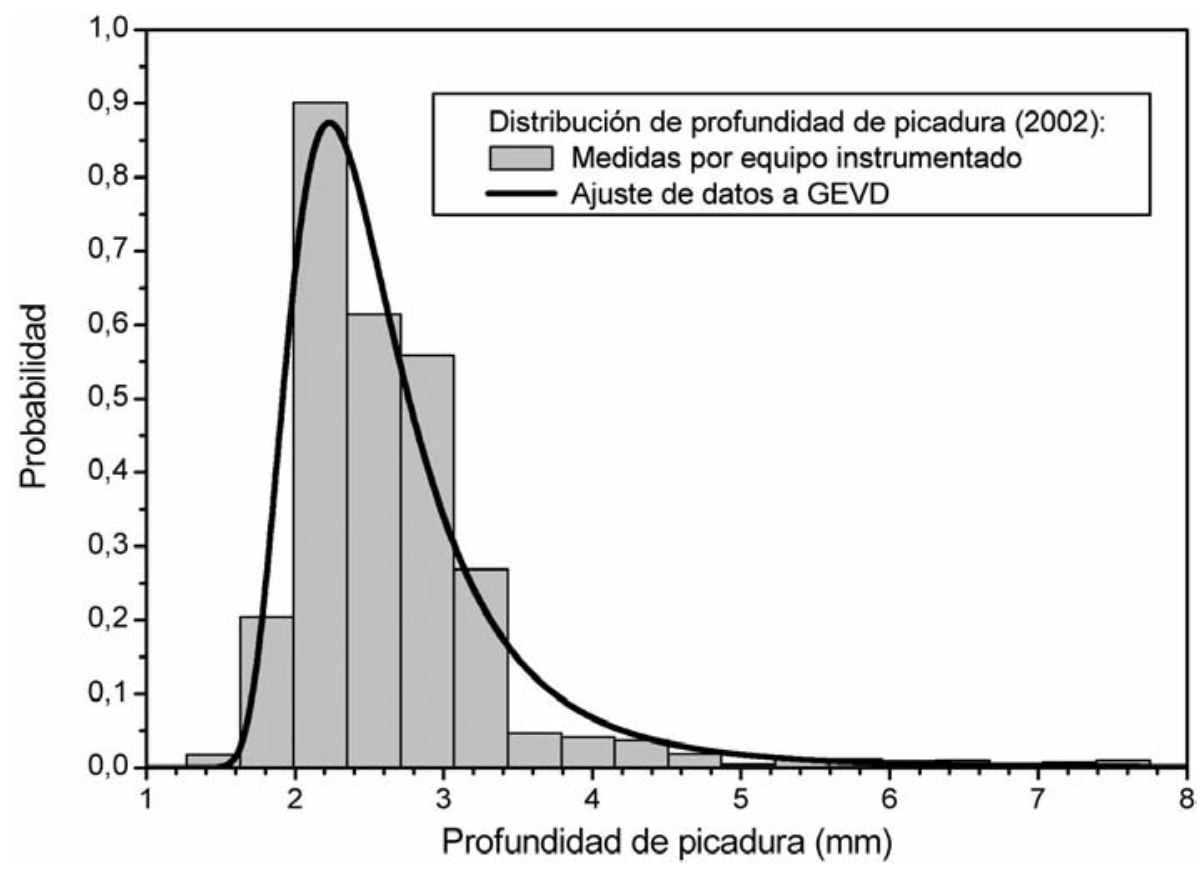

Figura 12. Distribución experimental y ajustada de profundidad de picadura en 2002.

Figure 12. Distribution Fit to the observed pit depth distribution in the studied pipeline in 2002.

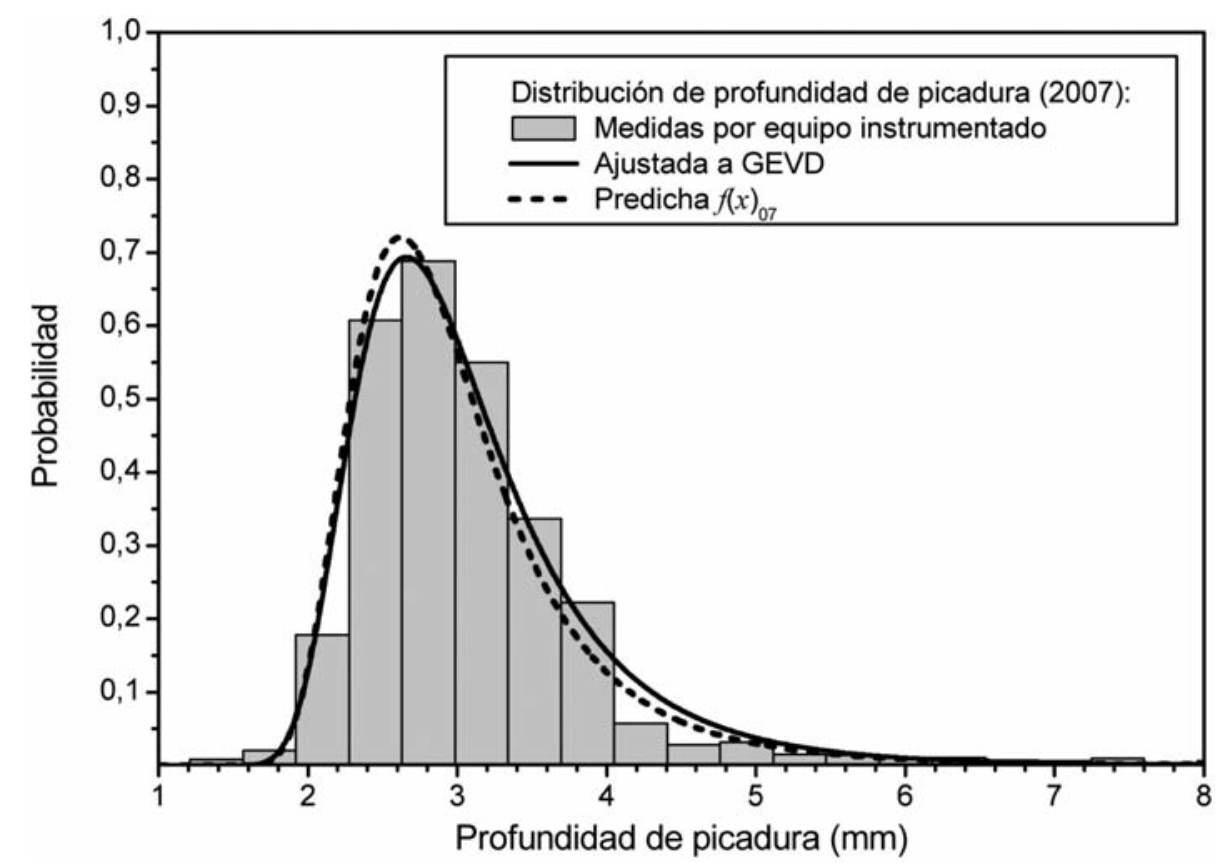

Figura 13. Distribución experimental y predicha de la profundidad de picadura en 2007.

Figure 13. Observed and predicted pit depth distribution in 2007.

Donde $\delta$, es el tiempo transcurrido entre las dos inspecciones (5,5 años) y el subíndice "il", indica que las profundidades de picadura fueron medidas en línea con equipo instrumentado. 
La función de probabilidad predicha que describe la profundidad de defectos para el año 2007, se muestra en la figura 13. En esta figura se puede observar que esta función describe correctamente la distribución empírica medida en campo. Esto indica que la suposición hecha respecto a las propiedades estadísticas de la velocidad de corrosión, es adecuada para este ducto.

Posteriormente, la ecuación (11) fue utilizada para estimar la evolución en el tiempo de la confiabilidad de ductos atribuible, a la función de velocidad de crecimiento $g(v)_{t}$. La distribución de profundidades medidas con equipo instrumentado en 2002, fue utilizada como distribución inicial y la confiabilidad fue estimada sobre un periodo de 15 años.

\section{MODELACIÓN ESTOCÁSTICA}

Uno de los elementos que hace posible modelar estocásticamente el crecimiento de picaduras en ductos, es el hecho de que es posible discretizar el espesor de pared de un tubo. Otra característica es que el proceso de corrosión por picadura, no presenta memoria del pasado.

Como definición general, se asume que el espesor de pared del tubo, se puede dividir en $\mathrm{N}$ estados y que la profundidad de picadura en un instante de tiempo $t$, puede ser representada por una variable aleatoria discreta $D(t)$ con $P\{D(t)=i\}=p_{i}(t)$; $i=1,2, \ldots, N$.

Además, se puede asumir que la probabilidad de que la profundidad en el estado $i$, avance un estado durante un intervalo corto de tiempo $t$, puede ser escrita como $\lambda_{i}(t) \delta t+o(\delta t)$. La probabilidad de que, para un tiempo $t$, el daño avance del estado $i$ al estado $j(j \quad i)$, en un intervalo $\Delta t$, puede obtenerse si se resuelve el sistema de ecuaciones diferenciales de Kolmogorov mostrado en las expresiones (12).

$$
\begin{aligned}
& \frac{\mathrm{d} p_{\mathrm{ij}}(\mathrm{t})}{\mathrm{dt}}=-\lambda_{\mathrm{j}}(\mathrm{t}) p_{i j}(\mathrm{t})+\lambda_{\mathrm{j}-1}(\mathrm{t}) p_{i j-1}(\mathrm{t}) \\
& \frac{\mathrm{d} p_{\mathrm{ij}}(\mathrm{t})}{\mathrm{dt}}=-\lambda_{\mathrm{i}}(\mathrm{t}) p_{i i}(\mathrm{t})
\end{aligned}
$$

Para un proceso de Markov, definido por las expresiones (12), es de interés encontrar la probabilidad de transición del estado $m$, al estado $n\left(\begin{array}{ll}n & m\end{array}\right)$ en el intervalo $\left(t_{0}, t\right)$, es decir, la solución $p_{m n}\left(t, t_{0}\right)$ del sistema 12 , se muestra a continuación y su existencia se demuestra en la referencia ${ }^{[17]}$, página 304.

$$
p_{m n}\left(t_{0}, t\right)=\left(\begin{array}{c}
n-1 \\
n-m
\end{array}\right) p s^{m}(1-p s)^{n-m}
$$

Donde:

$$
p s=e^{-\left\{\rho(t)-\rho\left(t_{s}\right)\right\}}
$$

y

$$
\rho(t)=\int_{0}^{t} \lambda(\tau) d \tau
$$

Analizando la ecuación 13, se puede afirmar que el incremento de la profundidad de picadura $(m-n)$, en un intervalo de tiempo $t-t_{0}$, corresponde a una distribución binomial negativa NegBin $(r, p)$, con parámetros $r=m$ y $p=p$ s. Cuando para $t=t_{i}$, el estado inicial es $n_{i}, d\left(t_{i}\right)=n_{i}$, la media estocástica del proceso $\bar{M}(t)=E[d(t)])$, está definida por la siguiente expresión ${ }^{[18]}$

$$
\bar{M}(\mathrm{t})=\mathrm{n}_{\mathrm{i}} \mathrm{e}^{\rho\left(\mathrm{t}-\mathrm{t}_{\mathrm{i}}\right)}
$$

Los autores Cox y Miller ${ }^{[18]}$, demuestran que la media determinista en algunos eventos, puede igualarse a la media estocástica del proceso de daño. En la presente investigación la ley determina que expresa el crecimiento de picaduras es la siguiente:

$$
\overline{\mathrm{D}}(\mathrm{t})=\mathrm{k}\left(\mathrm{t}-\mathrm{t}_{\mathrm{sd}}\right)^{v}
$$

Donde $t_{\mathrm{sd}}$ es el tiempo promedio de iniciación de picaduras, " $k$ " y " $v$ " son los parámetros de proporcionalidad y el exponente de la ley de crecimiento de defectos, respectivamente.

En el presente trabajo, se asume que la media experimental determinista para la profundidad de picadura $\overline{\mathrm{D}}(\mathrm{t})$, es igual a la media estocástica del proceso:

$$
\bar{D}(t)=\bar{M}(t)
$$

Si en la expresión 16 , se hace $n_{i}=1$; y el tiempo que el sistema consume en este estado inicial, es significativamente pequeño respecto al intervalo de tiempo del experimento, se puede demostrar que el valor de la función $\rho(t)$, se puede aproximar de la siguiente manera:

$$
\rho(\mathrm{t})=\ln \left(\mathrm{k}\left(\mathrm{t}-\mathrm{t}_{\mathrm{sd}}\right)\right)^{v}
$$

Y que la probabilidad ps, puede expresarse de la siguiente forma:

$$
p s=\left(\frac{t_{0}-t_{s d}}{t-t_{s d}}\right)^{v}
$$


Entonces, la probabilidad de transición de un estado $m$ a un estado $n$, en un intervalo de tiempo que va de $t$ a $t_{0}$, puede ser determinada a través de:

$$
p_{m n}\left(t_{0}, t\right)=\left(\begin{array}{c}
n-1 \\
n-m
\end{array}\right)\left(\frac{t_{0}-t_{s d}}{t-t_{s d}}\right)\left(1-\left(\frac{t_{0}-t_{s d}}{t-t_{s d}}\right)^{v}\right)^{n-m}
$$

La solución del sistema de ecuaciones diferenciales de Kolmogorov, solo depende del parámetro v, y del tiempo de iniciación de picadura $t_{\mathrm{sd}}$. Supongamos que la distribución de profundidad de defectos en un tiempo $t_{0}, P\left\{D\left(t_{0}\right)=m\right\}=p_{m}\left(t_{0}\right)$, es conocida. Por ejemplo, esta distribución puede ser obtenida mediante equipo instrumentado. En este caso, $t_{0}$ es el tiempo en que se llevó a cabo la inspección y el valor de las probabilidades $p_{m}$, estaría determinado por la relación del número de defectos encontrados en cada estado, respecto al número total de defectos. Si la función de probabilidad de transición $p_{m n}\left(t_{0}, t\right)$ se conoce, entonces es posible determinar la distribución de profundidad de defectos para cualquier tiempo futuro, utilizando la siguiente expresión ${ }^{[15]}$ :

$$
p_{\mathrm{n}}(\mathrm{t})=\sum_{\mathrm{m}=1}^{\mathrm{n}} p_{\mathrm{m}}\left(\mathrm{t}_{0}\right) p_{\mathrm{mn}}\left(\mathrm{t}_{0}, \mathrm{t}\right)
$$

Analizando la expresión anterior, se puede decir que la distribución de probabilidad de la profundidad de defectos en un tiempo $t$, es función de la distribución de probabilidad de profundidad de defectos en un tiempo $t_{0}$, y de la probabilidad de transición entre estados.

Esta última puede ser completamente determinada, si se conoce $(t)$, la cual es función directa de los parámetros $v$ y $t_{s d}$, en la expresión 16.

La figura 14, ilustra la evolución en el tiempo de la probabilidad ps. Analizando esta figura se puede observar lo siguiente:

- El valor de ps, disminuye conforme se incrementa la corrosividad del suelo.

- Para un mismo tipo de suelo aumenta el valor de ps si se aumenta $t_{0}$.

- Si se incrementa el intervalo de tiempo $t-t_{0}$, se observa una disminución de ps.

\subsection{Modelación markoviana de la profundidad de picadura en ductos}

A continuación, se expone como ejemplo de aplicación, el modelo desarrollado mediante cadenas de Markov, utilizando la información obtenida, mediante la inspección en línea mostrada en la sección

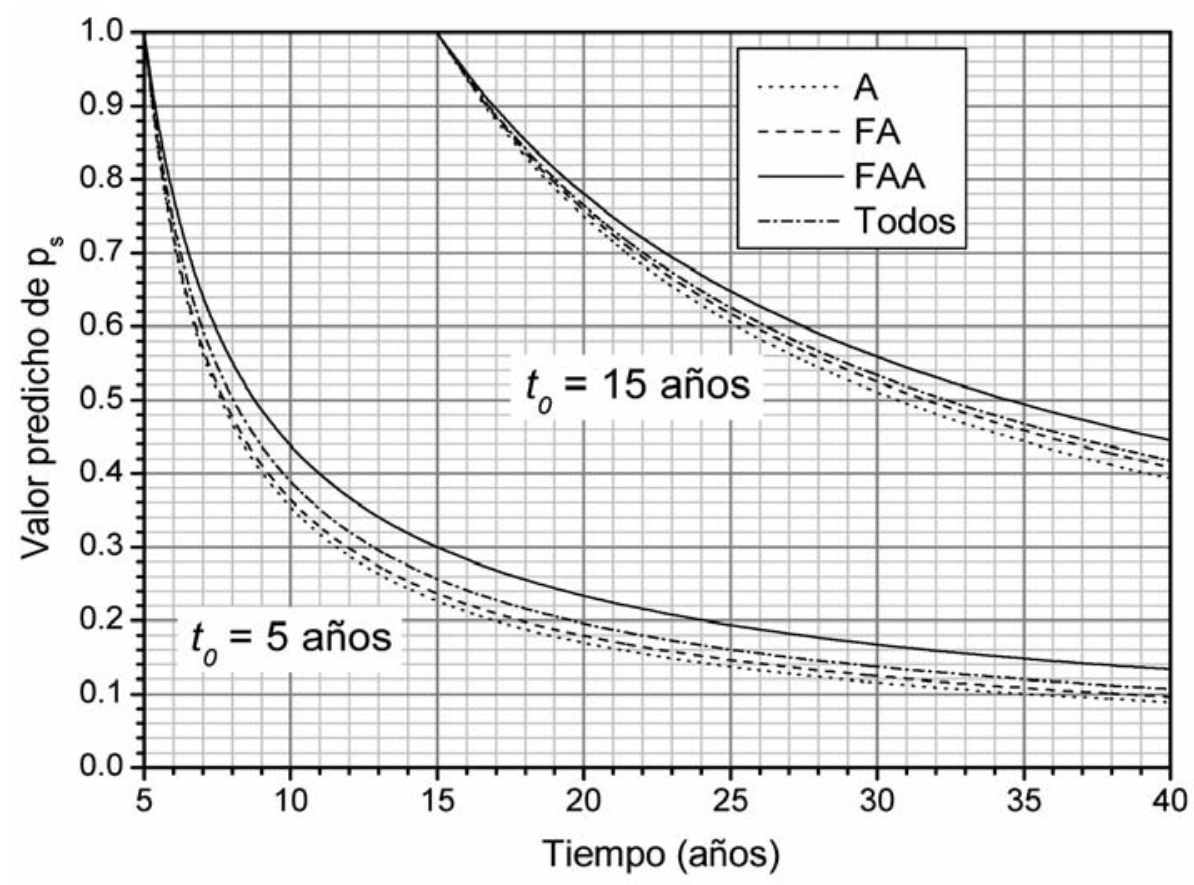

Figura 14. Evolución en el tiempo de ps por cada tipo de suelo, considerando $t_{0}$ de 5 y 15 años.

Figure 14. Evolution of ps for each soil category, considering $t_{0}=5$ and $t_{0}=15$ years. 
anterior. Los datos de inspección, se muestran en la figura 15. El histograma de barras achuradas, muestra la distribución de profundidad de picadura en 2002, con un número total de $N_{02}=3577$, defectos causados por corrosión externa localizada. El histograma mostrado en gris, representa los $N_{07}=3851$, defectos de corrosión medidos en 2007.

Para aplicar el modelo markoviano, la distribución de profundidades de defectos observada en 2002, fue utilizada como distribución inicial. De esta forma $t_{0}=21$ años, $t=5,5$ años y $p_{m}\left(t_{0}=21\right)=N_{m} / N_{02}$; siendo $N_{m}$ el número de defectos encontrados en el estado $m$. Se asumió, que las características del suelo a lo largo del ducto eran similares a las características presentadas en la categoría todos los suelos. Utilizando las expresiones 20 y 21 , se puede obtener la distribución de profundidad de defectos, producida por la modelación mediante cadenas de Markov. El resultado de esta modelación está representado en la figura por una línea continua. En la figura 15, también se puede observar la similitud existente entre la distribución observada en 2007 y los resultados obtenidos por la modelación markoviana. Indicando que este modelo de crecimiento de picadura, puede ser utilizado para determinar la evolución de la confiabilidad de ductos en el tiempo. Esto se debe al hecho, de que a partir de este tipo de modelación se puede determinar la velocidad de crecimiento de picadu- ras en función del tiempo, debido a que involucran la naturaleza estocástica de este fenómeno.

\section{CONCLUSIONES}

- Conforme al análisis de regresión realizado en la presente investigación, se puede decir que el parámetro de proporcionalidad $k$, de la ley de crecimiento de profundidad de picadura, es función del $\mathrm{pH}$, potencial redox, resistividad y contenido de iones del suelo, como se puede observar en la expresión 4. Por otra parte, el exponente $v$,está determinado por el tipo de recubrimiento, el potencial suelo tubo, la densidad aparente y el contenido de agua del suelo, tal y como se muestra en la expresión 5 .

- Entre las variables estudiadas, se puede afirmar que las de mayor influencia en el proceso de corrosión por picadura son: el valor de $\mathrm{pH}$, el potencial suelo - tubo, el tipo de recubrimiento del ducto, la densidad aparente, la humedad y la concentración de iones cloruro en el suelo.

- Dependiendo del tiempo, las distribuciones de valores extremos, Weibull, Gumbel o Fréchet, pueden ser consideradas como el mejor ajuste para los datos de profundidad de picadura y velocidad de crecimiento. Las distribuciones de Weibull y

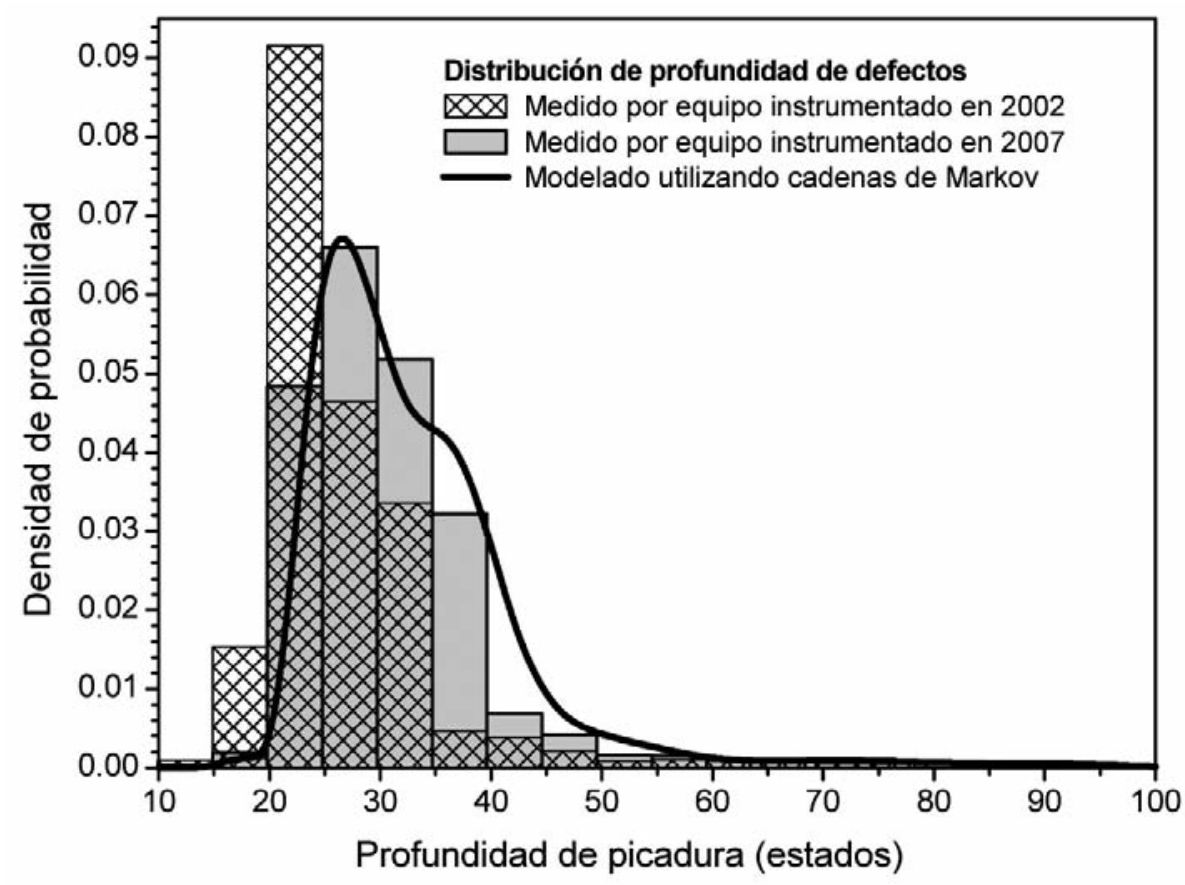

Figura 15. Resultados obtenidos por la modelación markoviana en un ducto en servicio.

Figure 15. Results of the Markov chain modeling in a pipeline. 
Gumbel, son las distribuciones que mejor se ajustan a los datos de profundidad de picadura y de velocidad de crecimiento para tiempos de exposición relativamente cortos $(<20$ años $)$. Para tiempos de exposición prolongados, la distribución de Fréchet llega a ser la mejor opción.

- Las distribuciones de velocidad de crecimiento de picadura desarrolladas en esta investigación, pueden ser utilizadas para estimar de manera realista, la evolución del crecimiento de picaduras en ductos en servicio.

- Es posible encontrar un nuevo modelo para determinar la profundidad de picadura en ductos enterrados, utilizando cadenas de Markov. El uso de un proceso de Markov no homogéneo, de nacimiento puro, para modelar el crecimiento de la corrosión por picaduras, es particularmente atractivo debido a la existencia de una solución analítica del sistema de ecuaciones diferenciales de Kolmogorov. El uso de esta solución, evita una reducción en el número de estados, lo que aumenta la simplicidad matemática.

- La probabilidad de transición de un estado m, a un estado $n$, solo es función del exponente de la ley de crecimiento de picaduras $v$, y del tiempo de iniciación de las misma $t_{\text {sd }}$.

- Los modelos propuestos, producen resultados satisfactorios en la estimación de vida útil de los ductos en servicio, logrando así una optimización en los tiempos y costos de mantenimiento.

\section{Agradecimientos}

J. C. Velázquez agradece al CONACYT de México por el apoyo económico para la realización de esta estancia postdoctoral en la Universidad de Delft.

\section{REFERENCIAS}

[1] M. Romanoff, Underground Corrosion, NBS Circular 579, NBS, Washington, EE.UU. D.C., (1979), pp. 50-70.
[2] J. Provan y E. Rodriguez, Corrosion Sci. 45 (1989) 178-192.

[3] A. Valor, D. Rivas, Caleyo F. y J.M. Hallen, Corrosion Sci. 49 (2007) 559-579.

[4] S. Bradford, Practical handbook of corrosion control in soils, CASTI Corrosion series, Edmonton, Canada, 2000, pp. 51-55.

[5] ASTM D422-63(2007) "Standard Test Method for Particle Size Analyses of Soils" (West Conshohocken, PA: ASTM International, 2007).

[6] A. Peabody, Control of pipeline corrosion, $2^{\mathrm{a}}$. Ed., R. Bianchetti, NACE International, Houston TX. EE.UU., 2001, pp. 30-40.

[7] S. Mughabghab y T. Sullivan, Waste Manage 9 (1989) 239-251.

[8] Y. Katano, H.Miyata y H. Shimizu, Corrosion, 59 (2003) 155-161.

[9] S. Papavinasam, R.W. Revie, NACE International Annual Corrosion Conf. and Exp. San Diego, CA, EE.UU. 2006, Paper No. 06047.

[10] J.Race, S. Dawson, L. Stanley y S. Kariyawasam, J. Pipe Eng. 6 (2007) 15-29.

[11] F. Kajiyama e Y. Koyoma, Corrosion Sci. 53 (1997) 156-162.

[12] M. Kowaka, H. Tsuge, M. Akashi y K. Masamura, An introduction to the life prediction of plant materials. Maruzen Pub. Tokio Japón, 1984, pp. 22-37.

[13] H.P. Hong, Corrosion Sci. 55 (1999) 10-15.

[14] J. Gentle, Random number generation and Monte Carlo Methods, 2를 Ed. Springer, New York, EE.UU. 2003, pp. 18-24.

[15] E. Castillo, A. Hadi, N. Balakrisjnan y J. Sarabia, Extreme Value and Related Models with Applications in Engineering and Science, WileyInterscience, New Jersey, EE.UU., 2005, pp. 21-55.

[16] E. Oguzie, L. Agochukwu y A. Onuchukwu, Mater. Chem. Phys. 84 (2004) 1-6.

[17] E. Parzen, Stochastic Process, SIAM Series, Oackland, California, EE.UU, 1999, pp. 304.

[18] D. Cox y H. Miller, The theory of stochastic processes, $1^{\underline{a}}$ Ed., Chapman \& Hall, CRC, Boca Raton, Florida, EE.UU., 1965, pp. 82. 